\title{
Adequacy of lymph node yield and staging in rectal cancer should not be determined based on a minimum number of lymph nodes evaluated
}

\author{
F. N. van Erning $・$ V. E. P. P. Lemmens
}

Accepted: 22 February 2015 / Published online: 8 March 2015

(C) Springer-Verlag Berlin Heidelberg 2015

\section{Dear Editor:}

With interest we read the paper 'A minimum yield of twelve lymph nodes in rectal cancer remains valid in the era of neoadjuvant treatment' by Lykke et al. published on 5 February 2015 [Epub ahead of print]. In this study, the authors found an association between increased lymph node yield and the detection of node positive disease up to a lymph node yield of 12 to 17 nodes for rectal cancer patients (irrespective of neoadjuvant treatment). They reason that this indicates that a yield of at least 12 lymph nodes is needed to ensure node negative disease, and that guidelines on a minimum yield of 12 lymph nodes are valid.

Although we agree with the authors that an adequate lymph node evaluation is important for correct staging and subsequent treatment of patients, we do not believe that their conclusion is justified that the adequacy of lymph node yield and staging can be determined on the basis of a certain minimum number of lymph nodes evaluated. In our opinion, certain important aspects were not taken into account in this study.

In the last two decades, the number of lymph nodes evaluated has become a surrogate marker for surgical and pathological excellence in colorectal cancer. Quality initiatives aimed at improving nodal yield have been undertaken and have resulted in an increase in the number of lymph nodes evaluated over time, indicating that room for improvement in surgical

F. N. van Erning $(\bowtie) \cdot$ V. E. P. P. Lemmens

Department of Research, Netherlands Comprehensive Cancer

Organisation (IKNL), Eindhoven, The Netherlands

e-mail: f.vanerning@iknl.nl

F. N. van Erning $\cdot$ V. E. P. P. Lemmens

Department of Public Health, Erasmus University Medical Centre,

Rotterdam, The Netherlands and pathological practices existed. However, a ceiling may have been reached beyond which further improvement might not be possible. This can be explained by the fact that the number of lymph nodes available for evaluation is not only influenced by the thoroughness of the surgeon and the diligence of the pathologist but also by a patient's ability to mount an effective immune response to the tumour. A growing body of literature (i.e. studies by Pages and Galon et al. in the New England Journal of Medicine, Science and Journal of Clinical Oncology) is showing that the biological behaviour of the tumour and host, such as immune response, also affects the number of traceable lymph nodes. A smaller number of lymph nodes reflects a diminished immune response and is also associated with less prominent lymphocytic infiltration into the primary tumour. Consequently, it may be unrealistic to only define a lymph node evaluation as adequate in case 12 or more lymph nodes are yielded.

Additionally, other recent studies among colon and rectal cancer patients such as the study by Parsons et al. in the Journal of the American Medical Association in 2011 and the study by Van Erning et al. in European Journal of Cancer in 2014 show that despite increases in lymph node yield over time, the proportion of lymph node positivity has remained unchanged, which makes one question the presumed understaging mechanism.

Therefore, we believe that adequacy of lymph node yield and staging in rectal cancer should not be determined based on a minimum number of lymph nodes evaluated. Surgeons and pathologists should strive to remove and evaluate all lymph nodes in the resection specimen, while acknowledging that fewer than 12 lymph nodes might be present in some patients. The absolute number of lymph nodes evaluated should not be used as a marker of quality but should rather be regarded as a reflection of immune response. 